\title{
The Properties and Applications of Quasicrystals
}

\section{Janot}

Institut Laue-Langevin, Grenoble, France

\begin{abstract}
In being relatively poor conductors of heat and electricity, quasicrystalline alloys, most of which contain about 70 atomic percent of aluminium, do not behave as one might expect. Other interesting properties involving, for instance, adhesion, corrosion, friction, and hardness, suggest that the industrial future of quasicrystals is promising while the present is already bearing its fruits, notably in coating applications.
\end{abstract}

Quasicrystals are materials having a new type of long-range order such that their diffraction patterns show Bragg reflections revealing symmetries which are incompatible with periodicity [1]. However, they are highly ordered systems [2] with correlation lengths of several tenths of a micrometer [3]. Large single crystals can be grown [4] whose quality is such that dynamical diffraction has been observed [3]. The atomic structure of quasicrystals can be reasonably well described in terms of a self-similar packing of atomic clusters using the techniques of highdimensional crystallography $[5,6]$.

Aside from the peculiar structures, quasicrystals also exhibit very unexpected properties [7]. Perhaps their most intriguing feature is a very high electrical (and thermal) resistivity. Its value, which almost equals that of an insulator [8], is unexpected for a material containing about 70 atomic percent of aluminium. Fundamental physical characteristics of this type combined with other unusual properties mean that quasicrystals have emerged in a surprisingly short period of time since their discovery as rather useful materials having valuable existing and potential applications.

\section{Very Different Structures}

Differences between the crystallography of periodic crystals (PC) and quasicrystals $(\mathrm{QC})$ can be examined in either real or reciprocal space. Both viewpoints are useful for understanding some of the properties of quasicrystals. To illustrate

Christian Janot is a Professor with the Université Joseph Fourier, Grenoble, and is based at the Institut Laue-Langevin, BP 156, F-38042 Grenoble Cedex, for his research work. He trained as a physical metallurgist and is presently involved in defining the materials research programme for the CNRS in France. schematically the real-space models of PC and QC, consider linear (one-

dimensional) chains built up with

sequences of two segments, one large (L) and one short (S). PC can be obtained via addition rules. For instance, adding strips LS over and over again gives the periodic chain LS LS LS LS LS ...... To obtain a QC chain, substitution rules must be used instead. For instance, any given strip of LS segments can be grown by substituting $L$ by $L S$ and $S$ by $L$ in sequence; this results in the following successively grown strips: - initial strip : L S - plus 1 substitution : L S L

2 substitutions : L S L L S 3 substitutions : L S L L S L S L etc.

The result is a perfectly ordered, deterministic sequence of $\mathrm{L}$ and $\mathrm{S}$ segments without any indication of periodicity. For onedimensional structures, it may be difficult to imagine why this awkward substitutional operation should be preferred instead of a straightforward periodic packing. Indeed, one-dimensional QC are expected to be unstable with respect to PC. But in two and three dimensions, substitution may be the only way to grow a structure. This is the situation, for instance, with pentagonal and icosahedral structural tiles (patterns of atoms), whose fivefold symmetries cannot be accommodated by periodicity. QC consequently become the stable structures as long as pentagonal or icosahedral atomic blocks can form.

In a monatomic PC such as a metal crystal, all atomic sites are strictly equivalent. If some electrons are loosely bonded to atoms they have no reason to locate on a particular site and can travel essentially freely through the bulk of the metal. The result is a high conductivity and isotropy, with all the well-known consequences for properties and their applications.
Conversely, in QC structures strictly equivalent sites cannot be found if the fully extended surroundings of the sites are considered. The "free" electrons, if there are any, are forced to "locate" preferably at low-energy sites within the constraints of the Coulomb interaction, although in reality this model must be somewhat smoothed out. QC structures are such that identical atomic clusters of any size can be found at distances apart of about twice the cluster size (Fig. 1). Conductivity is therefore reduced to a hopping or/and tunnelling communication between a hierarchy of almost identical sites, with bonding isotropy reduced to the preferred direction for hopping [9].

The reciprocal space description of QC adds to our understanding and supports similar conclusions. Both $\mathrm{PC}$ and QC structures can be analyzed in terms of their Fourier components in that the space dependence of their density can be expressed as a sum of density waves, i.e.,

$$
\rho(r)=(1 / v) \sum_{G} \rho(G) \exp (\mathrm{i} G . r) .
$$

For PC, the set of $G$ vectors define a discrete reciprocal lattice in which each wavevector can be written as an integer linear combination of three basis vectors $\boldsymbol{a}_{i}^{*}$ :

$$
G=h \boldsymbol{a}_{1}^{*}+k \mathrm{a}_{2}^{*}+\mathrm{la}_{3}^{*}
$$

For $\mathrm{QC}$, the number of integer linearly independent vectors required to span the reciprocal space exceeds the spatial dimension. For instance, six basis vectors are required to span the reciprocal space for three-dimensional icosahedral QC, i.e., Eq. (1) must be replaced by:

$$
\begin{aligned}
& \boldsymbol{G}= \\
& n_{1} \boldsymbol{a}_{1}^{*}+n_{2} \boldsymbol{a}_{2}^{*}+n_{3} \boldsymbol{a}_{3}^{*}+n_{4} \boldsymbol{a}_{4}^{*}+n_{5} \boldsymbol{a}_{5}^{*}+n_{6} \boldsymbol{a}_{6}^{*} .
\end{aligned}
$$

Many direct consequences follow:

- it is confirmed that the point symmetry is incompatible with periodic translational order in three-dimensions;

- the wavevectors $G$ define a set of points which fill the space densely;

- there is a periodic image of the structure in a higher dimensional space (six dimensions for icosahedral structures) which can been used in quasicrystallography approaches $[5,10]$.

The inferred differences between the properties of $\mathrm{PC}$ and $\mathrm{QC}$ are basically contained in the analysis of wave propagation in the two types of structures. Plane waves with any wavevector $k$ except for $k$ vectors which satisfy the diffraction condition,

$$
{ }_{2} G . k \pm|G|^{2}=0
$$


propagate easily in a periodic structure. The states that are excluded correspond to two-component standing waves; they remain extended but do not contribute to the propagation or transport of energy.

In $\mathrm{QC}$, the exception becomes the rule since the $G$-vectors form a dense set. In this case, any $k$-vector can comply with Eq. (2) and thus be diffracted. Moreover, it is readily demonstrated that multiple diffraction occurs generally and that the number of individual plane waves contributing to the resulting steady state increases with $|k|$. This comes from a simple geometrical derivation based on the Ewald sphere.

One is therefore tempted to ask if all propagation phenomena are excluded in QC. The basic question may be not this simple. Let us consider the particle (electron or phonon) associated with the plane wave of energy $\omega$ and wavevector $k$. In PC, the dispersion curve $\omega(k)$ shows a gap opening $\Delta \omega$ when $k$ obeys Bragg's law (Eq. 2 ) and has its extremity on the Brillouin zone limit. The lifetime of the particle is consequently reduced to $\Delta t \approx 1 / \Delta \omega$. Gap openings are in the $\mathrm{THz}$ range, i.e., $\Delta \omega \approx$ $10^{12} \mathrm{~Hz}$, for the phonon dispersion curve. For lifetimes of about $10^{-12} \mathrm{~s}$ and group velocities below $10^{3} \mathrm{~m} / \mathrm{sec}$, the corresponding free path is less than $10 \AA$. In a QC structure, the dispersion curve is actually a dense hierarchy of gaps; but most of the gaps are very narrow. With a typical value of $10^{8} \mathrm{~Hz}\left(10^{-4} \mathrm{Thz}\right)$ for $\Delta \omega$, the particle can propagate up to several micrometers. So propagative states can coexist with nonpropagative states in QC, the latter being much numerous and the former less effective than in crystals.

\section{Electrical and Thermal Conductivity}

In agreement with the analyses of the previous section, inelastic neutronscattering measurements $[9,11]$ show that extended real phonons are observed only in the vicinity of strong Bragg reflections for wavevectors smaller than $0.35 \AA^{-1}$ and energies below $1.5 \mathrm{THz}$ (equivalent to 6 $\mathrm{MeV}$ or about $70 \mathrm{~K})$. At higher energies, plane-wave modes are no longer relevant for describing atomic vibrations in QC. Energy scans at constant wavevector at points well removed from strong Bragg peaks result in a broad distribution of excitations (Fig. 2) which is very similar to the total "phonon" density of states (Fig. 3) [12]. Wavevector scans at constant high energy (e.g., at 3 and $5 \mathrm{THz}$ ) give flat dispersion curves - evidence for a lack of effective propagation. Interestingly, the minimum wavelength (or maximum wave-

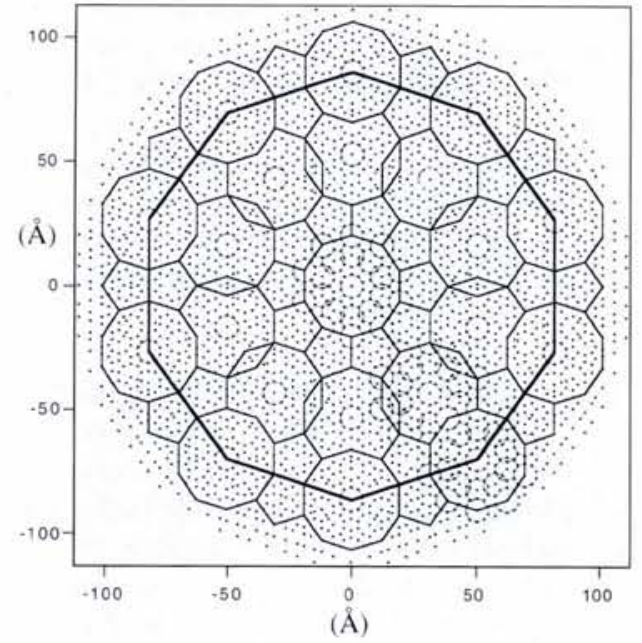

Fig 1. A planar section, perpendicular to a fivefold direction, through the structure of an icosahedral AlPdMn quasicrystal. Small rings of 10 atomic sites are visible. These correspond to the equatorial plane of the basic clusters. The rings are arranged in a selfsimilar ring of rings which, in turn, combine in the second inflation step to form a large decagon, and so on.

Fig. 2. Energy dispersion of the inelastic neutron scattering signal of an AlPdMn quasicrystal measured at two different values of the wavevectors at points in reciprocal space located away from any strong Bragg peak. Note the broad distribution of excitations.

vector) at which true unbroadened acoustic modes can be observed corresponds roughly to the largest standing wave that can settle into a cavity having the same size as the basic atomic unit in the structure $(\approx 9 \AA)$.

Such a reduced range of real extended phonons should result in very poor thermal conductivity, reaching a maximum value around $70 \mathrm{~K}$ when all possible phonons are fully excited (the Dulong-Petit regime) [9]. Most of these predictions are observed experimentally. The thermal conductivity values $K(T)$ are indeed very small - much smaller than those expected for purely metallic compounds. For instance, at room temperature, $K(T)$ for quasicrystalline AlFeCu and AlPdMn is more than two orders of magnitude smaller than for aluminum, more than one order of magnitude smaller than for steel, and about one-half that for zircon which is currently considered to be one of the best thermal insulators [13] (indeed, it is widely used because of its insulator properties). The phonon saturation effect is also observed as a plateau in the $K(T)$ curves covering a temperature range from about 25 to $100 \mathrm{~K}[14,15]$. The unexpected feature is that at higher temperature, $K(T)$ resumes an increasing trend (Fig. 4). This may be understood in terms of nonlinearity effects [9] which allow vibrational modes to interact. As a result, the high-energy recurrent standing modes, which can be dubbed as "clustrons" for simplicity, couple with the low-energy phonons. The net result is a "phononassisted clustron hopping" mechanism which contributes to a further increase of $K(T)$ [9].

For similar reasons, most of the electronic transport is expected to come from
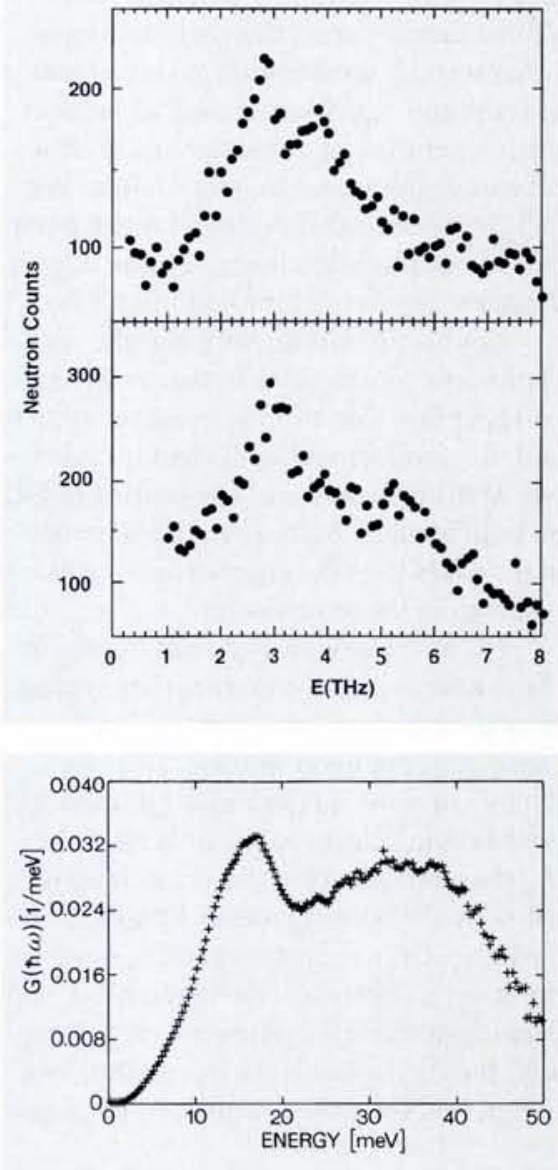

Fig. 3. The vibrational density of states for the icosahedral AIPdMn quasicrystal [12].

phonon-assisted collective electron hopping between equivalent sites in the structures, and QC must behave in almost the same way as insulators at low temperature, with recovery of some conductivity as the temperature increases. Such behaviour, based on a recurring hierarchical localization of the bonding electrons, is in fact in 


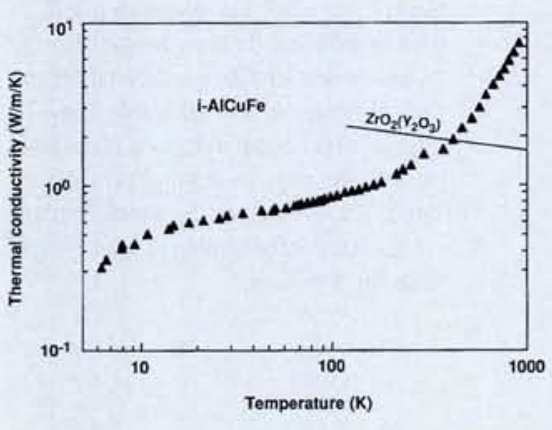

good agreement with what has been observed in resistivity data for the highest quality AlPdRe QC alloys [16]. At around $0.5 \mathrm{~K}$, the electrical resistivity of this QC can exceed $30 \Omega . \mathrm{cm}$, a value which is $10^{9}$ to $10^{10}$ times larger than that of pure aluminium (Fig. 5). The conductivity increases almost linearly upon heating, reaching a value at room temperature that is a factor of some 200-times larger than its value at the temperature of liquid helium. Chemical composition and quasicrystalline perfection are critical: defects and slight departures from ideal stoichiometry give a fairly dramatic recovery of the conductivity.

Application of this temperaturedependent conductivity in thermometry and heat flow detection is straightforward, and the development of devices is underway at the present time. The coating of QC on bulk metallic parts as an efficient thermal barrier layer for engines and the like is taken up in the next section.

QC alloys obviously do not belong to the conductor family; nor are they typical insulators since conductivity is significantly restored upon heating. Semiconductors or semi-metals cannot be considered as being closer to QC in as much as the electron density at the Fermi level in QC is smaller than in metals by a factor of only 3-10. QC definitely represent something quite different. The hierarchical packing of clusters in the structure along with the strong Coulomb interaction lead to repeated (so-called recurrent) localiza-
Fig. 4. The temperature dependence of the experimentally determined thermal conductivity of an AlFeCu icosahedral quasicrystal [15] compared with that of zircon.

tion of the bonding electrons. Phononassisted hopping conductivity may then generate "antipairing" in much the same way as the inverse (i.e., pairing) produces superconductivity.

\section{Other Properties and Applications}

The electrical behaviour analyzed in the previous section leads to class of related properties involving the Hall effect, thermal power, magnetic susceptibility, and optical conductivity. The negative value of the Hall coefficient $R_{\mathrm{H}}$ for the most perfect QC brings evidence that the majority of free carriers is made up of electrons [17]. Since the coefficient at room temperature is more than 10-times larger than for pure aluminum, there is a reduced density of free carriers. But as already stated, the reduction is not proportional to the amount expected on the basis of the low conductivity of QC. At the liquidhelium temperature of $4.2 \mathrm{~K}$ the negative $R_{\mathrm{H}}$ has decreased from about $-10^{-9}$ to $-10^{-8}$ which is again qualitatively, but not quantitatively, consistent with the temperature dependence of the conductivity.

Thermal power coefficients $S(T)$ for QC show an interesting behaviour [18]. They can be quite large, up to several tens of $\mu \mathrm{V} / \mathrm{K}$, are mostly negative in value and decrease down to a minimum at around $100 \mathrm{~K}$. Above room temperature they become increasingly positive. Tuning the temperature of the minimum seems to be possible using a small shift in composition and/or appropriate annealing treatments. This effect can be used to accurately monitor a temperature setting without the help of sophisticated electronic devices.

Magnetism also presents some curious features in QC. The presence of transition metals such as iron or manganese would normally introduce unpaired electron spins into the material, and paramag- netism, if not ferro- or antiferromagnetism, should be observed. This is not at all the case: strictly stoichiometric QC is diamagnetic, with a negative susceptibility of about $-5 \times 10^{-7} \mathrm{emu} / \mathrm{g}$. However, a transition from dia- to para-magnetism is observed at about $50^{\circ}$ below the melting point, and the susceptibility continues to increase with temperature in the liquid state [19] (Fig. 6). Diamagnetism occurs only when spins are exactly balanced. This happens for exactly saturated electronic states, as may be expected for stability of the recurrent localization in QC. Interestingly, QC exhibit some spin tunability via temperature or composition shifts which may be useful in electronic and computing devices. The frequency dependence $\sigma(\omega)$ of the electrical (or so-called optical) conductivity also deviates strongly from that for metallic behaviour $[20,21]$. In agreement with direct-current measurements, $\sigma(\omega)$ remains small for most of the frequency range, especially towards lowenergy values. However, a rather strong resonance shows up (Fig. 7) at around $10^{4}$ $\mathrm{cm}^{-1}$ or $290 \mathrm{THz}$ (corresponding to infrared radiation with a wavelength of about $1 \mathrm{~mm}$ ). Assuming this resonance comes from an electron-plasma oscillation, a mean-free path amplitude of about $22 \AA$ for the majority carrier can be deduced. Such a value is very close to the distance between two elementary clusters in the structure of AlPdMn QC (Fig. 1), something that would be expected if a hopping mechanism is relevant for conductivity in QC. Practical consequences should be very interesting, one of which is illustrated in Fig. 8 [22] showing that sandwiches of QC between oxide layers such as $\mathrm{SiO}_{2}$ or $\mathrm{Al}_{2} \mathrm{O}_{3}$ have a reflectivity $(R)$ gap centred on the visible wavelength range. A straightforward application of such a property involves energy absorbing/trapping coatings for solar cells, insulator screens or window glass.

A second class of QC properties are less well understood but interesting and

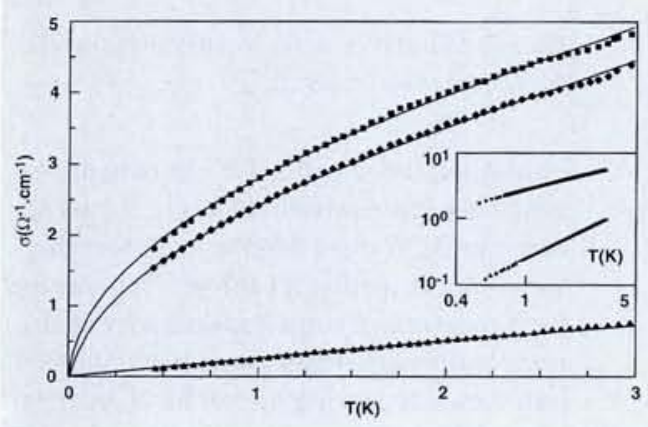

Fig. 5. The electrical conductivity $\sigma(T)$ at low temperatures of AIPdRe quasicrystals showing the $\sigma \sim T$ behaviour for a perfect icosahedral phase (lower curve) as well as the $\sigma \sim T^{0.5}$ law for a slightly less good sample (two uppermost curves). A $\log -\log$ plot is shown in the insert for the uppermost and lower curves [16].

Fig. 6. The magnetic susceptibility of an icosahedral quasicrystal demonstrating reversability with temperature [20].

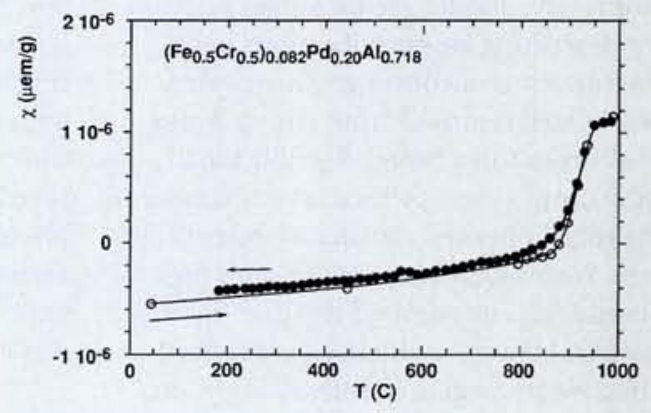




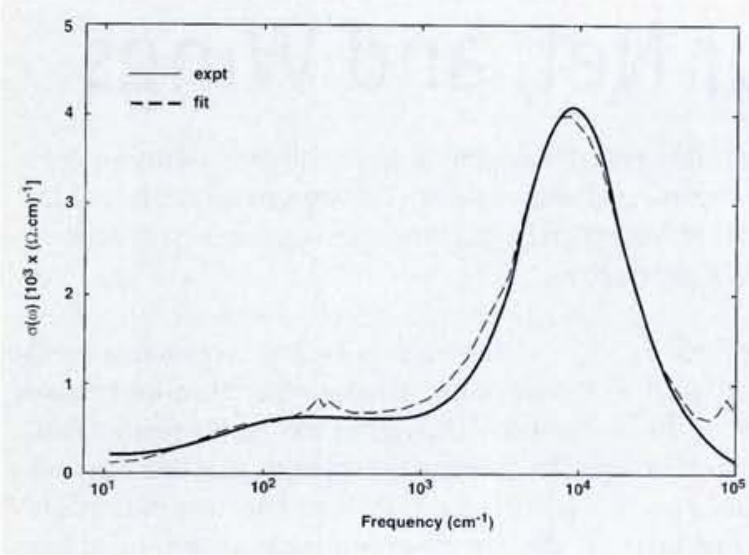

Fig. 7. The experimentally determined and curve-fitted optical conductivity $\sigma(\omega)$ of an icosahedral AIPdMn quasicrystal at room temperature as a function of the frequency $\omega$ [21].

Fig. 8. The reflectivity of a quasicrystal layer sandwiched between two layers of three different types of oxide insulators [22]. useful nonetheless, especially when some of them can be applied in combination. The class includes reduced wetting, a low friction coefficient, high hardness, elevated corrosion resistance, a ductile-brittle transition, and superplasticity above about $700^{\circ} \mathrm{C}$ (for most practical QC). High hardness is actually one of the strong points of QC. Values up to $10 \mathrm{GPa}$ have been measured [23], comparing favourably with the hardness levels for heat treated, tribological steels (so-called high-speed steels and steels used for ball bearings). Unfortunately, QC are very brittle at room temperature, with fracture strains as small as $0.35 \%$, i.e., fracture at strains that are only just above the value of $0.2 \%$ that is usually taken to be the engineering yield strain [24]. This restricts mechanical applications to coating situations, even though superplasticity shows up at high temperature with strains reaching up to $10 \%$ prior to fracture.

The wetting behaviour of QC surfaces lies at the origin of two very important properties, namely anti-adhesion [25] and low friction coefficients in tribological applications [26]. But as has been well known for a long time, there is no such thing as an intrinsically good friction behaviour because the friction partner, the third body (lubricant, wear debris, transfer layer, etc.) and testing conditions make their own contributions to the overall friction response. Published data [27] remain scarce and correspond mainly to dry scratch testing using various indenters with spherical tips. The friction coefficient with diamond remains as low as 0.05 under constant-load operations, for loading both below and above the critical value for transverse cracks to form. Friction coefficients for cemented carbide, hardened steel and alumina tips are of the order of 0.20 . Brittleness is attenuated within the scratch and in all cases, repeated passes reduce the friction coefficient down to a limit. QC friction on QC is being tested and seems to give coefficient almost as low as that obtained with diamond indenters.

Regarding corrosion resistance, QC compare quite well with stainless steel: their performance is ranked between austenitic and ferritic steels [28]. Surface analyses [29] seem to demonstrate that oxygen adsorbed on the surface of QC single crystals destroys quasiperiodicity within a layer of less than $20 \AA$ thick which passivates the material. A special, and highly interesting, aspect of the corrosion resistance of QC is their biocompatibility [30]. Measurements of samples implanted in living animals demonstrate the absence of chemical and immune reactions along with a long-duration tolerance.

Most of these properties combine effectively to give technologically interesting applications which have been protected recently by several patents [31]. For instance, the combination of non-sticking, hardness, corrosion resistance, and low thermal conductivity gives the almost ideal material for coating frying pans or other cookware: cooked food does not stick to the pan, which can be cleaned vigorously without damage; the food is not spoiled by chemical reaction and the liquid calefaction layer does not form thus improving greatly the cooking quality (for meat, in particular, but also for tarte Tatin, the French apple pie).

Quasicrystalline coatings, usually 10 $\mu \mathrm{m}$ to $1 \mathrm{~mm}$ in thickness, are made by plasma spraying powders produced by gas atomisation of a melt. Cost largely depends on the alloy composition, with coatings based on AlFeCu costing about 40 ECU $/ \mathrm{kg}$. Quasicrystalline powders are presently manufactured under the tradename CRISTOME for coating cooking utensils. Nearly 9 MECU is being

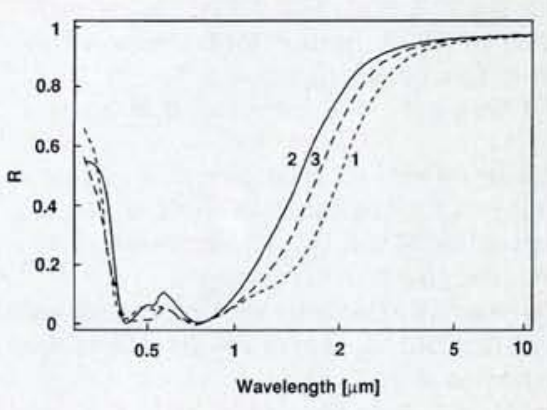

invested in the development with the aim of coating 10 million frying pans each year using about 500 tonnes of powder.

As a second example, combining the low friction coefficient and the high hardness and corrosion resistance is obviously very efficient in reducing both surface damage and energy dissipation in the moving contact between two solids. Quasicrystalline cylinder liners and piston coatings in motor-car engines would undoubtedly result in reduced fuel and lubricant consumption, reduced air pollution and increased engine lifetimes. The same set of associated properties (hardness, low friction, corrosion resistance) combined with biocompatibilty is also very promising for introducing QC in surgical applications as a coating on metallic parts used for bone repair and prostheses.

Finally, low thermal conductivity and corrosion resistance become especially useful at high temperatures when quasicrystalline coatings are superplastic. They constitute thermal screens that can easily accommodate thermal constraints and the thermal expansion of the protected bulk material, features that cannot be realized with today's best insulators such as zircon. Rocket motors and aeroengine turbines would benefit from this technology.

Quasicrystals are clearly fascinating materials: crystal structures, with their five-fold symmetry, are unconventional and properties are surprising and could be remarkably useful. Of course, reality may drop cold water here and there. For instance, producing industrial quantities of good quality quasicrystals is not that simple, although not impossible [31]. Material cost is another challenge, and traditionalism in industry has to be taken seriously. But the future is promising and the present is already bearing its fruits.

\section{References}

[1] Janot C., in Quasicrystals: A. Primer

(Oxford University Press) 2nd Edn., 1994. 
[2] Tsai A.P., et al., Mat. Trans. JIM 31 (1990) 98; Tsai A.P., A. Inoue \& T. Masumoto, Phil. Mag. Lett. 62 (1990) 95.

[3] Kycia S.W., et al., Phys. Rev. B 48 (1993) 3544 .

[4] de Boissieu M., et al., Phil. Mag Lett. 65 (1992) 147; Yokoyama Y., et al., Mater. Trans., Japan Inst. Metals 33 (1992) 97; Boudard M., et al., Phil. Mag Lett. 71 (1995) 11.

[5] Boudard M., et al., Phil. Mag. Lett. 64 (1991) 197; Boudard M., et al., J. Phys.: Condens. Matter 4 (1992) 149.

[6] Janot C. \& de Boissieu M., Phys. Rev. Lett. 72 (1994) 1674.

[7] Chernikov M.A., et al., Europhys. Lett. 21 (1993) 767; Pierce F.S., Poon S.J. \& Guo Q., Science 261 (1993) 737; Chernikov M.A., et al., Phys. Rev. B 48 (1993) 3058; Klein T., et al., Phys. Rev. Lett. 66 (1991) 2907; [15] Lanco P., et al., Europhys. Lett. 18 (1992) 227; Mayou D., et al.,Phys. Rev. Lett. 70 (1993) 3915.

[8] Poon S. J., Adv. Phys. 41 (1992) 303.

[9] Janot C., Phys. Rev. 53 (1996) 1.

[10] Cornier-Quiquandon M., et al., Phys. Rev. B 44 (1991) 2071.

[11] Boudard M., et al., J. Phys.: Condens. Matter 7 (1995) 7299; and Phys. Scripta T57 (1995) 84.

[12] Suck J. B., J. Non-Cryst Solids 153-154 (1993) 573.

[13] Dubois J.M., et al., J. Mater. Res. 73 (1992) 38.

[14] Chernikov M.A., Bianchi A. \& Ott H.R., Phys. Rev. B 51 (1995) 153; Perrot A. \& Dubois J.M., Ann. Chim. Fr. 18 (1993) 501.

[15] Perrot A. \& Dubois J.M., Proc. 6th Int. Conf. on Quasicrystals, Eds.: C. Janot \& R. Mosseri (World Scientific, 1995) 586.

[16] Pierce F.S., Guo Q. \& Poon S.J., Phys. Rev. Lett. 73 (1994) 2220.

[17] Lanco P., PhD Thesis, Univ. Joseph Fourier, Grenoble (1993).

[18] Biggs B.D., Poon S.J. \& Munirathman N.R., Phys. Rev. Lett. 65 (1990) 2700.

[19] Boursier D., et al., C.R. Acad. Sci. Paris 319 II (1994) 409.

[20] Degiorgi L., et al., Solid State Comm. 87 (1993) 721.

[21] Basov D.N. et al., Phys. Rev. Lett. 73 (1994) 1865.

[22] Eisenhammer T., in [15] 766.

[23] Kang S.S. \& Dubois J.M., Phil. Mag A 66 (1992) 161; Dubois J.M., Kang S.S. \& Perrot A., Mater. Sci. Engng. A 179-180 (1994)122.

[24] Kang S.S. \& Dubois J.M., Europhys. Lett. 18 (1992) 46.

[25] Dubois J.M., et al., Ann. Chim. Fr. 19

(1994) 3 .

[26] Kang S.S., Dubois J.M. \& von Stebut J., J.

Mater. Res. 8 (1993) 2471.

[27] Von Stebut J., Strobel C. \& Dubois J.M., in [15] 702.

[28] Massiani Y., Ait-Yaazza S. \& Dubois J.M. in $[15] 788$.

[29] Chang S.L., et al., Surface Sci. 337 (1995) 135.

[30] Sedel L., Janot C. \& Dubois J.M. (to be published).

[31] For information, contact: J.M. Dubois, LSG2M, Ecole des Mines, Parc de Saurupt, F54042 Nancy Cedex.

\section{Spread Your Net, and Wings}

Denis Weaire, Professor of Natural and Experimental Philosophy at Trinity College, Dublin, who steps down shortly as the Chairman of the Physics Panel for the Research Training Networks activity of the European Union's Training and Mobility of Researchers Programme, argues that physics networks need to pay more attention to industrial participation.

All over Europe, the research networks supported by the Human Capital and Mobility (HCM) Programme of the European Union (EU) are filing their final reports and closing their accounts. For many individuals this was their first taste of science on a European scale, and they like it. Sceptics have said that the programme's main beneficiaries were the airlines, but those closest to the ground know that it has undoubtedly quickened the pace of international collaboration.

HCM was never intended to scatter its funds as widely and thinly as it did. The tortuous negotiations which preceded it resulted in compromises which left its description so vague as to defy interpretation at some points. Even the panels which were called to select the successful proposals were unsure of their mission and priorities. Accordingly, in gambling parlance, they played the field.

Proposers were disgruntled to receive news of grants below their expectation levels. Since then, many of them have come to appreciate the benefits of marginal funding to facilitate cooperation. Is this not a particularly appropriate role for the EU ?

The original idea of strong, well funded networks was reinstated in the successor to HCM. This was dubbed the Training and Mobility of Researchers (TMR) Programme, as the European Commission pursued its relentless search in the dictionary for new names. Lower as well as upper bounds on funding levels were fixed so that the eventual grants would lie in the range of 1-2 MECU per network. The tendency of panels to throw bread upon the waters was to be held in check. Criteria for selection were clear. The race was now on.

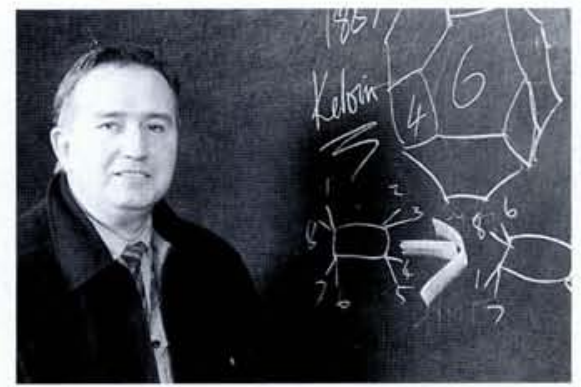

The eventual $6.4 \%$ success rate for the first round of network applications caused much dismay, but was surely predictable. To alleviate the repetition of this orgy of ritual rejection, the allocation of funds to the next two rounds have been rolled into one, and the deadline deferred until 16 September 1996. The success rate should roughly double, but the competition will still be tough. Is it worth applying?

Rounding up a flock of network partners and getting them all to point in the same direction is no easy task. While the proposal forms (first gate in the sheepdog trial) have been greatly improved, they still pose puzzles for the uninitiated. Many will rightly conclude that it is more realistic and efficient to try to attract individual TMR Research Training Grants - that part of the programme had an average success rate of close to $25 \%$ for postdoc fellowships in its first two rounds (physicists amounted to approximately $22 \%$ of applicants, and achieved a satisfactory $24 \%$ success rate).

If you do decide to conceive a prospective network (or dust off an old one) you should bear in mind that all three words training/mobility/research must be respected. In the end, having covered all the bases, remember that selection is by scientific peer review. The peers involved will have spent three days in Brussels scrutinising the proposals, discussing their merits, and consuming mussels. As selection fatigue sets in they will look for exciting and challenging themes, startling originality, potential for progress and strong interactions of all kinds: theory with experiment, country with country, big centres of excellence with small ones.

You should think hard about the possible involvement of industry. TMR is neutral as regards basic and applied science. By default it has become a major morale booster for basic science, but its future would be better assured if it were more balanced in this respect.

Physics presently suffers a particular malaise in its relations with industry,

D.L. Weaire, was elected as the EPS President-Elect at the EPS Council in Lisbon on 22-23 March. 